\title{
Effect of the emphysema subtypes of patients with chronic obstructive pulmonary disease on airway inflammation and COTE index
}

\author{
ZHENG LIU ${ }^{1}$, FANG SHI ${ }^{1}$, JUN-XIA LIU ${ }^{1}$, CHANG-LAN GAO ${ }^{1}$, MENG-MIAO PEI ${ }^{1}$, JING LI ${ }^{1}$ and PEI-XIU LI ${ }^{2}$ \\ Departments of ${ }^{1}$ Respiratory Tract Medicine and ${ }^{2}$ Radiology, The Petroleum Clinical Medical \\ College of Hebei Medical University, Langfang, Hebei 065000, P.R. China
}

Received October 18, 2017; Accepted July 13, 2018

DOI: $10.3892 /$ etm.2018.6799

\begin{abstract}
The aim of the present study was investigate the association of the severity of emphysema of patients with chronic obstructive pulmonary disease (COPD) with airway inflammation and the COPD-specific comorbidity test (COTE) index. A total of 94 patients with COPD were divided into four groups according to the severity of their emphysema; in each patient, comorbidities were recorded and inflammatory biomarkers, including MMP-9 and TIMP-1 were determined in circulating blood. The unbalanced proportion of MMP-9 and its inhibitor, TIMP-1, led to the airway inflammation and lung remodeling in the patients with COPD. A total of $80.85 \%$ of the patients had emphysema of different degrees. The quantity of male patients and the smoking index in the three emphysema groups were significantly higher than those in the non-emphysema group $(\mathrm{F}=7.67$ and 5.42, $\mathrm{P}<0.05)$. The level of the predicted percent offorced expiratory volume in $1 \mathrm{sec}$ in the non-emphysema group were significantly higher than those in the emphysema group (4.33; $\mathrm{P}<0.05)$, and the level of $\mathrm{D}$-dimer in the non-emphysema group was significantly lower than that in the mild and moderate emphysema groups $(\mathrm{F}=9.38, \mathrm{P}<0.05)$. The low-attenuation area score was negatively correlated with inhaled bronchodilators $(\mathrm{r}=-0.240, \mathrm{P}=0.007)$ but positively correlated with the frequency of acute exacerbations in the previous year $(\mathrm{r}=0.211, \mathrm{P}=0.001)$. In terms of treatment, the use of systemic hormone therapy in the emphysema group was more frequent than that in the non-emphysema group $(\mathrm{F}=6.21,12.92$ and $4.08, \mathrm{P}<0.05)$. The level of MMP-9 was significantly higher in COPD patients with $>3$ comorbidities, a COTE index of $\geq 4$ and cardiovascular disease as well as coronary heart disease $(\mathrm{t}=6.40,2.53,3.65$ and $2.90, \mathrm{P}<0.05)$. The level of MMP-9 was positively correlated
\end{abstract}

Correspondence to: Dr Pei-Xiu Li, Department of Radiology, The Petroleum Clinical Medical College of Hebei Medical University, 51 Xinkai Road, Langfang, Hebei 065000, P.R. China

E-mail: cnpeixiuli@126.com

Key words: chronic obstructive pulmonary disease, type of emphysema, airway inflammation, comorbidities with the neutrophilic granulocyte percentage, the number of comorbidities and the COTE index $(r=0.193,0.402$ and 0.311 , $\mathrm{P}<0.01)$. The severity of emphysema in patients with COPD was correlated with the persistence of inflammatory factors in the circulating blood and the frequency of acute exacerbations. It was indicated that MMP-9 has a critical role in numerous comorbidities of COPD.

\section{Introduction}

Individualized assessment and treatment of chronic obstructive pulmonary disease (COPD) are important in the management of this disease. However, the pathogenesis of COPD has remained to be fully elucidated and requires multiple measures to assess its severity, including the COPD Assessment test, lung function test, as well as the determination of the frequency of acute exacerbations, comorbidities, subtypes and a chest computed tomography (CT) scan (1-5). In addition, inflammatory cells and inflammatory mediators are involved in the inflammatory response and severe disorganization of the lung tissue structure; particularly metalloproteinases (MMPs) have a key role in the destruction of airway and lung tissues, as well as in airflow limitation, associated with COPD (6-8). Comorbidity is a major factor influencing the mortality of COPD patients. The COPD-specific comorbidity test (COTE) index has been utilized by Divo et al (9) to demonstrate that comorbidities of COPD patients were closely linked to the fatality rate. The present study aimed to assess the effect of the severity of emphysema in COPD patients determined by chest CT on airway inflammation, the level of MMPs in the circulating blood and a variety of common comorbidities. Such studies in this field are rare, and the present study is helpful in evaluating the overall clinical features of COPD patients, while aiding in the establishment of an index for the individualized treatment of patients with COPD.

\section{Patients and methods}

Study population. The present study was performed at the Petroleum Clinical Medical College of Hebei Medical University (Langfang, China). A total of 94 cases that presented at the hospital between January 2016 and 
December 2016 were enrolled in the study; of these, 56 cases were male and 38 were female, and their age ranged from 48 to 87 years. The mean age of the participants was $71.06 \pm 8.99$ years. The participants were divided into two groups: A and B. Group A contained 69 patients (73.4\%) who were current and former smokers, and the remaining 25 patients $(26.6 \%)$, who had never smoked, were assigned to group B. The diagnosis of COPD was based on the criteria established in a study by Celli and MacNee (10). The present study was authorized by the Ethics Committee of the Petroleum Clinical Medical College of Hebei Medical University. All patients who were enrolled in this study had provided their informed consent and had been examined for lung function 3 months prior to being hospitalized. In addition, they had undergone high-resolution CT (HRCT) of the chest. All 94 patients underwent routine blood examination; the levels of MMP-9 and its inhibitor, tissue inhibitor of metalloproteinases-1 (TIMP1) were measured on the second day after admission. All MMP-9 and TIMP-1 kits were purchased from Beijing Dongge Biotech Co., Ltd., Beijing, China. A total of $5 \mathrm{ml}$ of fasting venous blood was collected from all patients at 6:00-8:00 am using a test tube without pyrogen and endotoxin to enable sampling over $2 \mathrm{~h}$. Then, blood was centrifuged at a speed of $1,275 \mathrm{x} \mathrm{g}$ for $10 \mathrm{~min}$ at $37^{\circ} \mathrm{C}$. This separated serum from the blood. Subsequently, $100 \mu 1$ serum was packed into several test tubes. It was then stored at a temperature of $-80^{\circ} \mathrm{C}$ for later use. According to the Kitaguch method (11) and the score of the low-attenuation area (LAA), the emphysema of the 94 COPD patients was evaluated, and based on the results, 28 cases were assigned to group A (LAA, 4-6 points; mild emphysema group), eight cases to group B (LAA, 7-9 points; moderate emphysema group), 40 cases to group C (LAA, $\geq 10$ points; severe emphysema group) and 18 cases to group D (LAA, 0-3 points; non-emphysema group).

Chest HRCT examination. Prior to the examination, all 94 patients were taught the breath holding method for measuring the maximum end inspiratory and maximum end expiratory valuesand the examination was performed using a second generation spiral CT (Siemens AG, Munich, Germany); subsequently, they were trained to reach their best condition. The effective tube voltage was $120 \mathrm{kV}$, the tube current was auto-mAs, scanning collimation was $128 \times 0.6 \mathrm{~mm}$, the pitch of screw was 1.45 , the rotation time was $0.33 \mathrm{sec}$, the image scanning layer thickness was $5 \mathrm{~mm}$, the reconstruction layer thickness was $1 \mathrm{~mm}$ and the scanning ranged from the apex to the bottom of the lungs. The target scanning parameters were as follows: Scanning layer thickness, $1 \mathrm{~mm}$; interlamellar spacing, $0.5 \mathrm{~mm}$; a B70f algorithm and $512 \times 512$ matrix were used for rebuilding; the remaining parameters were the same as above.

Assessment of emphysema and bronchial wall thickness. The measures of emphysema were as follows: Emphysema was quantitatively measured using the HRCT visual subjective semi-quantitative method and by processing the thin layer $(1 \mathrm{~mm})$ CT image with Syngo CT Pulmo 3D image processing software (Siemens Healthcare, Munich, Germany), using an LAA of $<-960 \mathrm{HU}$. The following three anatomical layers were
Table I. Comorbidities and COTE index score.

\begin{tabular}{lcc}
\hline Comorbidities & Risk factor & COTE index \\
\hline Lung oresophageal cancer & $\geq 2$ & 6 \\
Pancreatic or breast cancer & & \\
Other tumor types & 13.76 & 6 \\
Anxietyor depression & 1.68 & 2 \\
Liver Cirrhosis & 1.56 & 2 \\
Atrial fibrillation & 1.54 & 2 \\
Diabetic neuropathy & 1.51 & 2 \\
Pulmonary interstitial fibrosis & 1.33 & 1 \\
Congestive heart failure & 1.32 & 1 \\
Gastric/duodenal ulcer & 1.28 & 1 \\
Coronary heart disease & & \\
\hline
\end{tabular}

COTE, chronic obstructive pulmonary disease-specific comorbidity test.

selected for observation, as presented in Fig. 1: The distance between the first anatomical layer and the upper margin of the aortic archwas $1 \mathrm{~cm}$ (Fig. 1A); the second anatomical layer was located $1 \mathrm{~cm}$ from the horizontal margin of the carina (Fig. 1B); and the other layer contained a distance of $3 \mathrm{~cm}$ to theupper margin of the right diaphragm (Fig. 1C). The LAA was determined for three lung fields based on the percentage of each layer in each lung field. The LAA score was calculated for each layer, as previously described (9): 0 points for LAA $<5 \%, 1$ point for $5 \% \leq \mathrm{LAA}<25 \%, 2$ points for $25 \% \leq \mathrm{LAA}<50 \%, 3$ points for $50 \% \leq \mathrm{LAA}<75 \%$ and 4 points for LAA $>75 \%$. The grading was based on the sum of the score of the three layers: Grade 0 for 0 points, grade 1 for 1-3 points, grade 2 for $4-6$ points, grade 3 for 7-9 points and grade 4 for $10-12$ points.

Following the determination of the cross-section perpendicular to the fifth bronchial lumen (as confirmed by two experience radiologists), the scanning images which assessed bronchial wall enlargement of the right upper lobe were used to rebuild the apical segmental bronchus with multiplanar reformation. Bronchial wall thickness (T) and pulmonary artery (PA) diameter in the same layer were measured using image magnification (x2.5) and the ratio of airway wall thickness to the pulmonary artery diameter (T/PA) was calculated. Bronchial wall thickness in 3 layers was classified as previously described (9) and were as follows: Grade 0 for $\mathrm{T} / \mathrm{PA}<30 \%$, Grade 1 for $30 \% \leq \mathrm{T} / \mathrm{PA}<50 \%$, and Grade 2 for $\mathrm{T} / \mathrm{PA} \geq 50 \%$.

The COTE index was determined on the basis of the scoring method designed by Divo et al (9). As presented in Table I, COTE $\geq 4$ points was determined in 14 cases and $0 \leq \mathrm{COTE} \leq 3$ points was determined in 80 cases.

Statistical analysis. Statistical analysis was performed with SPSS 19.0 software (IBM Corp., Armonk, NY, USA). Measurement data were analyzed by an independent-samples $\mathrm{t}$-test for comparison between two groups; analysis of variance followed by a least-significant differences post-hoc test was 


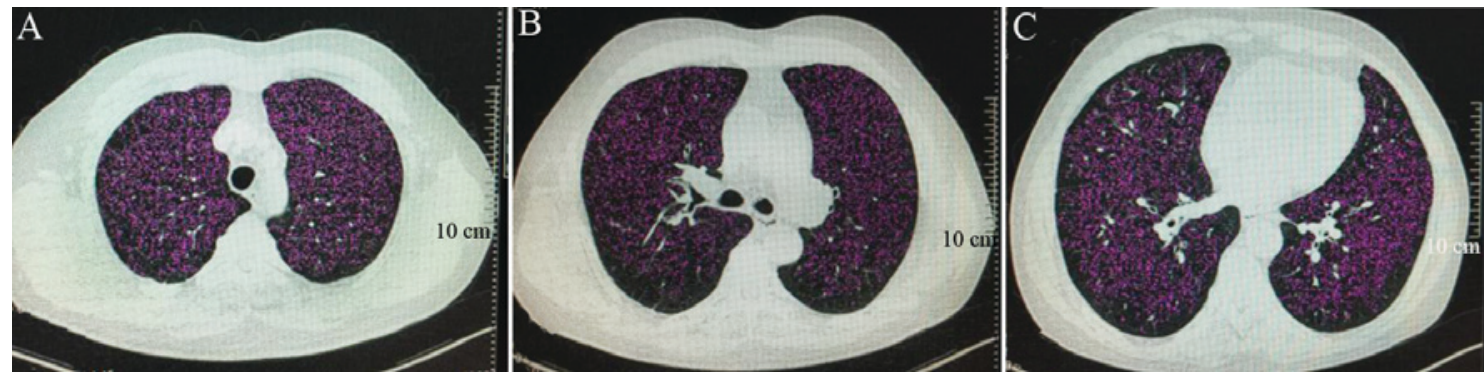

Figure 1. The distance between the first anatomical layer and the upper margin of the aortic archwas $1 \mathrm{~cm}$ (Fig. 1A); the second anatomical layer was located $1 \mathrm{~cm}$ from the horizontal margin of the carina (Fig. 1B); and the other layer contained a distance of $3 \mathrm{~cm}$ to theupper margin of the right diaphragm (Fig. 1C). The magenta part is the low attenuation area.

used for comparison among multiple groups. Enumeration data were analyzed using the chi-squared test. Correlations were analyzed by calculation of the Pearson correlation coefficient. Levene's test was used to determine the homogeneity of variance among the experimental groups. $\mathrm{P}<0.05$ was considered to indicate a statistically significant difference.

\section{Results}

General characteristics of COPD patients. The general characteristics of the cohort of COPD patients, whose major manifestation on the chest CT was emphysema, are presented in Table II and Fig. 1. The magenta areas in Fig. 1 represent the LAA of group C. The homogeneity of variance test (Levene's test) was used to analyze the data of each group and the results indicated no statistically significant difference among all groups $(\mathrm{P}>0.05)$. As presented in Table II, the percentages of males and patients with a smoking history in the three emphysema groups (group A, B and C) were significantly higher than those in the non-emphysema group $\mathrm{D}(\mathrm{F}=7.67$ and 5.42, respectively; $\mathrm{P}<0.05)$, and the percentage of patients with a history of smoking in the severe emphysema group was higher than that in the mild emphysema group. Within the cohort of COPD patients, female patients more commonly had no emphysema and no history of smoking. The proportion of one's full vital capacity of forced expiratory volume in $1 \mathrm{sec}$ (FEV1\% pred) in the non-emphysema group were significantly higher than those in the emphysema group $(4.33, \mathrm{P}<0.05)$. Furthermore, the level of $\mathrm{D}$-dimer in the non-emphysema group was significantly lower than that in the mild and moderate emphysema groups $(\mathrm{F}=9.38$, $\mathrm{P}<0.05)$. There was no statistically significant difference in patient age, neutrophilic granulocyte percentage (NEUT\%), the percentage of lymphocytes, eosinophilic granulocyte percentage, procalcitonin (PCT), residual volume/total lung capacity, maximum mean expiratory flow (MMEF) $25-75 \%$, N-terminal pro b-type natriuretic peptide (NT-proBNP), partial oxygen pressure $\left(\mathrm{PaO}_{2}\right)$ and TIMP-1 as well as MMP-9/TIMP-1 among three emphysema groups $(\mathrm{P}>0.05)$. In addition, there was no statistically significant difference in the airway thickness among the four groups $(\mathrm{P}>0.05)$.

Comorbidities and COTE index. The comorbidities, COTE index and glucocorticoid treatment of the COPD patients in all four groups are presented in Table III. There was no statistically significant difference in the number of comorbidities or the COTE index among the four groups $(\mathrm{P}>0.05)$. The number of COPD patients with pulmonary heart diseasein group A was significantly lower than that in the other three groups $(\mathrm{B}, \mathrm{C}$ and $\mathrm{D} ; \mathrm{P}<0.05)$. Furthermore, the patients in group $\mathrm{D}$ were significantly more likely to have cardiovascular disease as a comorbidity than those in the other three groups $(\mathrm{P}<0.05)$. Compared with group $\mathrm{C}$, a statistically significant difference in the number of COPD patients with cardiac insufficiency in groups D and A was identified $(\mathrm{P}<0.05)$. Regarding coronary heart disease as a comorbidity, the number of affected COPD patients in group A was significantly higher than that in group $\mathrm{C}(\mathrm{P}<0.05)$. In terms of intravenous glucocorticoid infusion, a significantly higher number of patients received this treatment in groups $\mathrm{A}, \mathrm{B}$ and $\mathrm{C}$, compared with those in group $\mathrm{D}(\mathrm{P}<0.05)$. In addition, there was no significant difference in the incidence of acute exacerbation and respiratory failure of patients with COPD among the four groups ( $\mathrm{P}>0.05$; Table III).

Comorbidities and the level of serum MMP-9. As presented in Table IV, there was a statistically significant difference in the expression of MMP-9 between the COPD patients who exhibited $>3$ comorbidities or a COTE index of $\geq 4$ points and the patients who exhibited fewer comorbidities $(t=6.40$ and 2.53, respectively; $\mathrm{P}<0.05)$. There was a statistically significant difference in the expression of MMP-9 between the COPD patients with either cardiovascular disease (hypertension, arrhythmia, coronary heart disease) or simple coronary heart disease and the patients without either cardiovascular disease or coronary heart disease $(\mathrm{t}=3.65$ and 2.90 , respectively; $\mathrm{P}<0.05)$. However, the levels of MMP-9 were not significantly affected by the presence of pulmonary heart disease, respiratory failure, cardiac insufficiency or the number of acute exacerbations in the previous year ( $\mathrm{P}>0.05$; Table IV).

Correlation analysis. A correlation analysis between CT types and clinical parameters indicated that the LAA score was negatively correlated with the MMEF25-75\% and treatment with inhaled bronchodilators $(\mathrm{r}=-0.148$ and -0.240 , respectively; $\mathrm{P}=0.044$ and 0.007 ); but positively correlated with the probability of $\geq 2$ acute exacerbations in the previous year $(\mathrm{r}=0.211, \mathrm{P}=0.001)$. The LAA grades were negatively correlated with inhaled bronchodilators $(\mathrm{r}=-0.280, \mathrm{P}=0.003)$. The correlation between MMP-9, TIMP-1 or MMP-9/TIMP-1 and clinical parameters was then assessed. The level of MMP-9 was positively correlated with the NEUT\%, the quantity of 
Table II. Comparison of the general condition in the 4 groups of patients.

\begin{tabular}{|c|c|c|c|c|c|c|}
\hline Groups & Group A $(n=28)$ & Group B $(n=8)$ & Group C $(n=40)$ & Group D $(n=18)$ & $\mathrm{F}$ & P-value \\
\hline Age & $70.50 \pm 7.99$ & $70.75 \pm 9.74$ & $71.75 \pm 8.93$ & $71.22 \pm 9.30$ & 0.14 & 0.95 \\
\hline Males & $17(18.1)$ & $6(6.4)$ & $31(32.9)$ & $2(2.1)^{\mathrm{a}-\mathrm{c}}$ & 7.67 & $<0.001$ \\
\hline Smokers & $19(67.8)$ & $7(87.5)^{\mathrm{a}}$ & $34(85.0)^{\mathrm{a}}$ & $9(50.0)^{\mathrm{a}-\mathrm{c}}$ & 5.42 & 0.49 \\
\hline NEUT\% $\%$ & $72.85 \pm 14.72$ & $76.52 \pm 12.86$ & $75.30 \pm 9.96$ & $73.81 \pm 12.67$ & 0.38 & 0.82 \\
\hline LYM\% & $18.37 \pm 13.11$ & $16.62 \pm 12.04$ & $16.80 \pm 8.12$ & $18.25 \pm 10.25$ & 0.29 & 0.92 \\
\hline $\mathrm{EO} \%$ & $0.84 \pm 1.01$ & $1.87 \pm 3.37$ & $0.99 \pm 1.86$ & $0.98 \pm 1.36$ & 1.17 & 0.53 \\
\hline PCT & $0.18 \pm 0.17$ & $0.32 \pm 0.19$ & $0.48 \pm 1.00$ & $0.39 \pm 0.97$ & 0.94 & 0.49 \\
\hline $\mathrm{RV} / \mathrm{TLC} \%$ & $51.83 \pm 7.98$ & $54.01 \pm 5.42$ & $51.49 \pm 9.10$ & $53.33 \pm 9.20$ & 0.15 & 0.80 \\
\hline MMEF25-75\% & $22.59 \pm 12.60$ & $21.94 \pm 15.75$ & $25.56 \pm 9.83$ & $17.26 \pm 10.90$ & 2.79 & 0.30 \\
\hline FEV1\% & $45.78 \pm 17.20$ & $41.34 \pm 15.47$ & $39.85 \pm 18.42$ & $48.28 \pm 20.23^{\mathrm{a}}$ & 4.33 & 0.04 \\
\hline NT-proBNP & $2301.57 \pm 2309.81$ & $1452.09 \pm 1387.52$ & $1722.99 \pm 2170.35$ & $2246.94 \pm 2616.80$ & 0.36 & 0.62 \\
\hline D-dimer & $1.74 \pm 1.84$ & $1.56 \pm 14.64$ & $1.34 \pm 1.23$ & $1.28 \pm 1.74^{\mathrm{a}, \mathrm{b}}$ & 9.38 & $<0.001$ \\
\hline $\mathrm{PaO}_{2}(\mathrm{mmHg})$ & $73.46 \pm 11.21$ & $78.67 \pm 7.71$ & $71.34 \pm 15.95$ & $71.09 \pm 16.66$ & 0.76 & 0.57 \\
\hline MMP-9 (ng/l) & $127.00 \pm 10.71$ & $128.53 \pm 7.07$ & $129.30 \pm 6.95$ & $139.34 \pm 11.43$ & 0.41 & 0.75 \\
\hline TIMP-1 (ng/l) & $118.43 \pm 16.89$ & $130.90 \pm 18.96$ & $118.98 \pm 15.76$ & $140.20 \pm 64.13$ & 2.60 & 0.08 \\
\hline MMP-9/TIMP-1 & $0.85 \pm 0.11$ & $0.84 \pm 0.14$ & $0.88 \pm 0.12$ & $0.84 \pm 0.16$ & 1.64 & 0.58 \\
\hline $\mathrm{T} / \mathrm{PA}$ & $0.38 \pm 0.11$ & $0.34 \pm 0.08$ & $0.36 \pm 0.09$ & $0.34 \pm 0.06$ & 0.99 & 0.42 \\
\hline
\end{tabular}

${ }^{a} \mathrm{P}<0.05$ vs. group $\mathrm{A} ;{ }^{b} \mathrm{P}<0.05$ vs. group $\mathrm{B} ;{ }^{\mathrm{C}} \mathrm{P}<0.05$ vs. group $\mathrm{C}$. Values are expressed as $\mathrm{n}(\%)$ or the mean \pm standard deviation. MMP-9, matrix metalloproteinase 9; TIMP-1, tissue inhibitor of metalloproteinases-1; FEV1\%, the proportion of one's vital capacity, measured in 1 second; EO\%, eosinophilic granulocyte percentage NEUT\%, neutrophilic granulocyte percentage; LYM\%, lymphocyte percentage; PCT, procalcitonin; RV/TLC, residual volume/total lung capacity; MMEF, maximum mean expiratory flow; NT-proBNP, N-terminal pro b-type natriuretic peptide; $\mathrm{PaO}_{2}$, partial oxygen pressure; T/PA, pulmonary aorta thickness.

Table III. Analysis of the comorbidities, COTE score and hormone intervention in the 4 groups.

\begin{tabular}{|c|c|c|c|c|c|c|}
\hline Groups & $\begin{array}{c}\text { Group A } \\
(\mathrm{n}=28)\end{array}$ & $\begin{array}{l}\text { Group B } \\
(\mathrm{n}=8)\end{array}$ & $\begin{array}{c}\text { Group C } \\
(\mathrm{n}=40)\end{array}$ & $\begin{array}{l}\text { Group D } \\
(\mathrm{n}=18)\end{array}$ & $\mathrm{F}$ & P-value \\
\hline Number range of comorbidities & $3.29 \pm 1.68$ & $1.88 \pm 1.36$ & $3.00 \pm 1.54$ & $3.06 \pm 1.66$ & 1.65 & 0.19 \\
\hline $\begin{array}{l}\text { Number range of patients with pulmonary } \\
\text { heart disease }\end{array}$ & $0.29 \pm 0.46$ & $0.00 \pm 0.00^{\mathrm{a}}$ & $0.15 \pm 0.36^{\mathrm{a}, \mathrm{b}}$ & $0.11 \pm 0.32^{\mathrm{a}, \mathrm{b}}$ & 9.15 & $<0.001$ \\
\hline Number range of patients with raspiratory failure & $1.29 \pm 0.46$ & $1.38 \pm 0.52$ & $1.25 \pm 0.44$ & $1.22 \pm 0.43$ & 0.90 & 0.86 \\
\hline $\begin{array}{l}\text { Number range of patients with cardiovascular } \\
\text { disease }\end{array}$ & $0.68 \pm 0.48$ & $0.38 \pm 0.52$ & $0.68 \pm 0.47$ & $0.89 \pm 0.32^{\mathrm{a}-\mathrm{c}}$ & 19.9 & $<0.001$ \\
\hline $\begin{array}{l}\text { Number range of patients with coronary heart } \\
\text { disease }\end{array}$ & $0.21 \pm 0.42$ & $0.25 \pm 0.46$ & $0.35 \pm 0.48^{\mathrm{a}}$ & $0.33 \pm 0.49$ & 3.34 & 0.06 \\
\hline Number range of patients with cardiac insufficiency & $0.32 \pm 0.48^{c}$ & $0.25 \pm 0.46$ & $0.13 \pm 0.34$ & $0.28 \pm 0.46^{c}$ & 5.16 & 0.04 \\
\hline $\begin{array}{l}\text { Number range of patients with intravenous } \\
\text { hormone therapy }\end{array}$ & $1.07 \pm 0.26$ & $1.13 \pm 0.35$ & $1.05 \pm 0.22$ & $1.00 \pm 0.00^{\mathrm{a}-\mathrm{c}}$ & 6.21 & 0.02 \\
\hline $\begin{array}{l}\text { Number range of acute exacerbations in the } \\
\text { previous year, } \geq 2\end{array}$ & $1.54 \pm 1.04$ & $1.13 \pm 0.35$ & $1.54 \pm 1.04$ & $1.56 \pm 1.19$ & 2.63 & 0.11 \\
\hline Range of COTE score & $2.32 \pm 2.42$ & $0.50 \pm 0.93$ & $2.03 \pm 2.46$ & $1.28 \pm 1.26$ & 1.03 & 0.21 \\
\hline
\end{tabular}

${ }^{\mathrm{a}} \mathrm{F}=6.21-33.58, \mathrm{P}<0.05$ compared with group $\mathrm{A} ;{ }^{\mathrm{b}} \mathrm{t}=4.82-12.92, \mathrm{P}<0.05$ compared with group $\mathrm{B} ;{ }^{\mathrm{c}} \mathrm{t}=4.08-18.81, \mathrm{P}<0.05$ compared with group $\mathrm{C}$. Values are expressed as the mean \pm standard deviation. COTE, chronic obstructive pulmonary disease-specific comorbidity test.

comorbidities, the COTE index and cardiovascular disease as well as coronary heart disease $(r=0.193,0.402,0.351$, 0.309 and 0.219 ; all, $\mathrm{P}<0.05)$. The expression of TIMP-1 and PCT were positively correlated with inhaled bronchodilators ( $r=0.149$ and $0.170 ; P=0.045$ and 0.044 , respectively). The
MMP-9/TIMP-1 ratio was negatively correlated with inhaled bronchodilators $(r=-0.175, \mathrm{P}=0.039)$. Further correlation analysis of comorbidities and clinical parameters revealed that the COTE index was positively correlated with the NT-proBNP, PCT, application of intravenous hormone therapy and MMP-9 
Table IV. Association of comorbidities and COTE index with the expression of the MMP-9 and TIMP-1.

\begin{tabular}{|c|c|c|c|c|}
\hline Parameter & $\mathrm{N}$ & MMP-9 (ng/l) & TIMP-1 (ng/l) & P-value \\
\hline \multicolumn{5}{|c|}{ Number of comorbidities } \\
\hline $0-3$ & $55(58.5 \%)$ & $124.34 \pm 7.22$ & $120.73 \pm 21.95$ & 0.26 \\
\hline$>3$ & $39(41.5 \%)$ & $134.50 \pm 8.08^{\mathrm{a}}$ & $128.35 \pm 42.75$ & $<0.001$ \\
\hline \multicolumn{5}{|c|}{ COTE score } \\
\hline $0-3$ & 80 & $127.59 \pm 8.79$ & $125.60 \pm 34.03$ & 0.37 \\
\hline$\geq 4$ & 14 & $134.06 \pm 9.04^{\mathrm{a}}$ & $114.15 \pm 16.98$ & $<0.001$ \\
\hline \multicolumn{5}{|c|}{ Pulmonary heart disease } \\
\hline No & $78(82.9 \%)$ & $128.23 \pm 8.30$ & $124.39 \pm 33.49$ & 0.44 \\
\hline Yes & $16(17.1 \%)$ & $130.16 \pm 12.38$ & $121.48 \pm 26.18$ & 0.75 \\
\hline \multicolumn{5}{|c|}{ Respiratory failure } \\
\hline No & $69(73.4 \%)$ & $128.57 \pm 9.40$ & $124.81 \pm 36.90$ & 0.98 \\
\hline Yes & $25(26.6 \%)$ & $128.51 \pm 8.29$ & $121.37 \pm 12.87$ & 0.65 \\
\hline \multicolumn{5}{|c|}{ Cardiovascular disease } \\
\hline No & $29(30.9 \%)$ & $123.76 \pm 7.66$ & $123.32 \pm 25.83$ & 0.91 \\
\hline Yes & $65(69.1 \%)$ & $130.70 \pm 8.88^{\mathrm{a}}$ & $124.15 \pm 34.93$ & $<0.001$ \\
\hline \multicolumn{5}{|c|}{ Coronary heart disease } \\
\hline No & $66(70.2 \%)$ & $126.86 \pm 8.47$ & $126.62 \pm 35.87$ & 0.21 \\
\hline Yes & $28(29.8 \%)$ & $132.57 \pm 9.33$ & $117.46 \pm 20.66$ & 0.01 \\
\hline \multicolumn{5}{|c|}{ Cardiac insufficiency } \\
\hline No & $73(77.7 \%)$ & $127.62 \pm 8.86$ & $124.09 \pm 34.58$ & 0.92 \\
\hline Yes & $21(22.3 \%)$ & $131.82 \pm 9.27$ & $123.23 \pm 23.13$ & 0.06 \\
\hline \multicolumn{5}{|c|}{ Acute exacerbations in the last year } \\
\hline $0-1$ & $50(53.2 \%)$ & $127.21 \pm 9.28$ & $129.47 \pm 40.26$ & 0.55 \\
\hline$\geq 2$ & $44(46.8 \%)$ & $130.09 \pm 8.67$ & $117.56 \pm 18.07$ & 0.34 \\
\hline
\end{tabular}

${ }^{a} \mathrm{t}=2.53-6.40, \mathrm{P}<0.05$ compared with ' $0-3$ ' or ' $\mathrm{no}$ '. Values are expressed as the mean \pm standard deviation. COTE, chronic obstructive pulmonary disease-specific comorbidity test; MMP, matrix metalloproteinase; TIMP, tissue inhibitor of metalloproteinases.
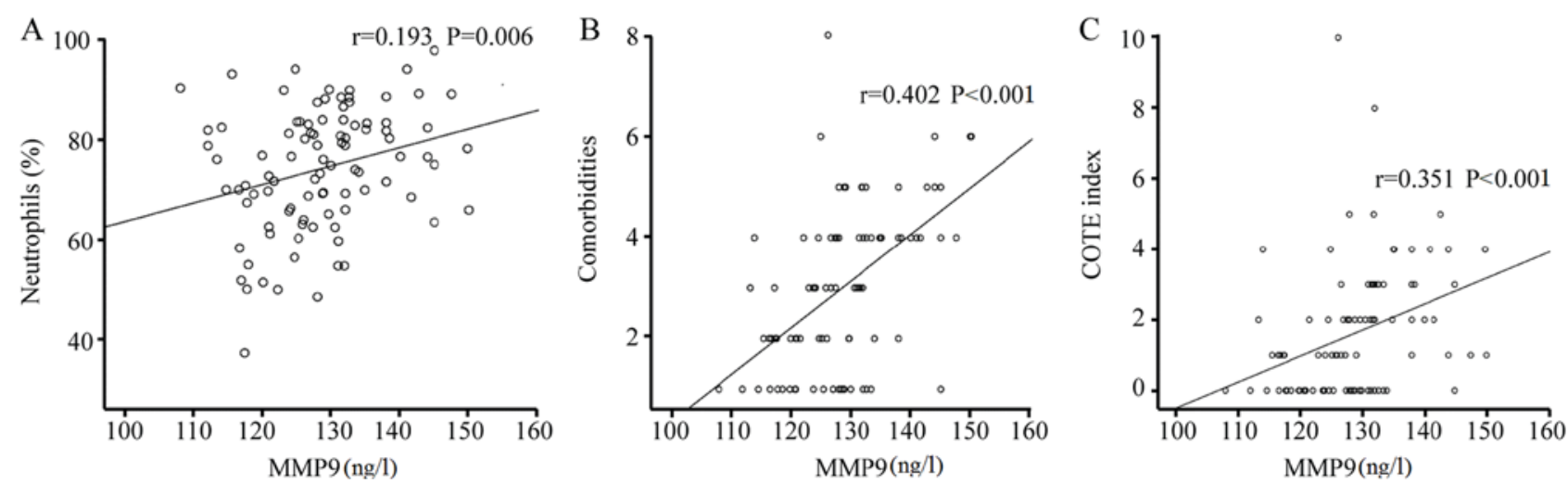

Figure 2. (A) Correlation between MMP-9 and the percentage of neutrophils; (B) number of comorbidities; (C) COTE index in subjects with COPD. COPD, chronic obstructive pulmonary disease; c, COTE index; MMP-9, matrix metalloproteinase 9; COTE, COPD-specific comorbidity test.

$(\mathrm{r}=0.455,0.166,0.227$, and $0.351 ; \mathrm{P}<0.001, \mathrm{P}=0.031,0.015$ and $<0.001$, respectively). Analysis of the correlation between the NT-proBNP and clinical parameters then indicated that the NT-proBNP was positively correlated with the NEUT\%, intravenous hormone therapy, COTE index and the number of comorbidities ( $\mathrm{r}=0.177,0.183,0.264$ and $0.410 ; \mathrm{P}=0.012,0.032$, 0.002 and $<0.001$, respectively; Fig. 2).

\section{Discussion}

The study of COPD is an important aspect in the field of chronic airway diseases. In the clinic, accurate evaluation of COPD is helpful for the individualized treatment of patients. Additional assessments that are of high importance include the lung function test, recording of clinical symptoms, acute 

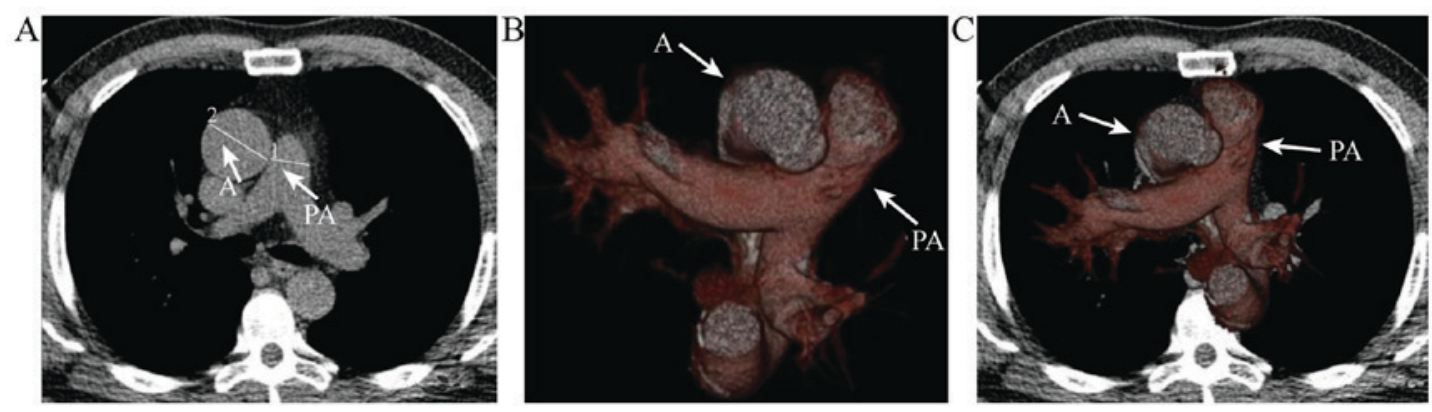

Figure 3. Measurement of the pulmonary artery to aorta ratio in chest CT scans. (A) If A was not uniform in diameter, two measurements were taken 90 degrees apart and the larger diameter was utilized. 1 indicates the diameter of PA, 2 indicates the diameter of A. (B) Digital three-dimensional reconstruction in axial cross section. (C) the three-dimensional reconstruction overlaid on the axial CT image. CT, computed tomography; PA, pulmonary artery; A, artery.

episodes and symptomatic phenotypes of COPD, determination of the area of emphysema and the cross-sectional area ratio of the pulmonary artery to the aorta, the 6-min walking test and the COTE index. In its essence, COPD is a chronic inflammation in the small airways with subsequent formation of emphysema (12). The HRCT may be used for macroscopic identification of the severity of chronic airway inflammation and emphysema $(13,14)$. The present study aimed to assess the effect of the destruction of the lung structure on the airway inflammation, airflow limitation and comorbidities, primarily from the perspective of the classification of emphysema within the HRCT of COPD patients; thus, it provides a fundamental theory for individualized treatment.

In the present study, 94 patients with COPD were divided into four groups using the Kitaguch method, which was regardless of whether thickening of the small airway was present, but according to the LAA score and the severity of emphysema. The sample size in group B was significantly lower than that in the other three groups. Thus, it was verified that eachsample size among the four groups was not significantly different $(\mathrm{P}>0.05)$ by using the homogeneity of variance test, revealing that the sample size in the four groups was comparable. It was identified that $80.85 \%$ of COPD patients (76/94) exhibited emphysema at various degrees, while $20 \%$ of patients were free of emphysema. There were a variety of risk factors, including smoking and air pollution. These factors may cause small airway stenosis and destruction of the lung parenchyma (15); furthermore, they may cause a decrease in the pulmonary elastic recoil of COPD patients, which leads to emphysema that is invoked by various inflammatory mediators, including MMP-9 (16-18).

Previous studies have indicated that centrilobular emphysema is the most common type of emphysema in smoking patients with COPD, particularly in the right upper lobe (19-21). The level of peripheral blood leukocytes increased more markedly in COPD patients who exhibited centrilobular emphysema and panlobular emphysema than in those with paraseptal emphysema. Furthermore, smoking was indicated to be a vital factor in increasing the severity of airway inflammation in patients with centrilobular emphysema.

The present study indicated that the proportion of patients with a history or current status of smoking in the emphysema groups was significantly higher than that in the non-emphysema group, and the number of smoking patients in the severe emphysema group was higher than that in the mild emphysema group, which indicated that the severity of emphysema in COPD patients was associated with airway inflammation caused by smoking.

MMP-9 is a proteolytic enzyme, which is dependent on zinc and calcium ions. It has an important role in the degradation of extracellular matrix (ECM) and cell membranes, thus causing enlargement of the alveolar spaces and a diminished elastic recoil of the alveoli, consequently leading to gas retention; in addition, MMP-9 is involved in the inflammatory response to airway and lung remodeling by activating inflammatory factors and destroying the epithelium or endothelial structure (22). TIMP-1 is an inhibitor of MMP-9; it inhibits the activation of the MMP-9 by blocking the zymogens activation of MMPs and specifically binds to zinc ions in the catalytic active center of MMP-9, consequently preventing the ECM from excessive degradation. The imbalance of MMP-9/TIMP-1 leads to airway inflammation and remodeling $(2,7,23)$. In the present study, the expression of MMP-9 in the COPD patients with mild emphysema was lower than that in those without emphysema, and there was no statistically significant difference in the expression of MMP-9 among the three emphysema groups $(\mathrm{P}>0.05)$. The level of TIMP-1 was also increased in the non-emphysema group compared with that in the three emphysema groups, but there was no statistically significant difference $(\mathrm{P}>0.05)$. This result was considered to indicate that MMP-9 has a key role in the pathogenesis of COPD, and in addition, MMP-9 is known to be involved in the pathogenesis of bronchitis. Of note, the MMP-9 levels were positively correlated with the frequency of acute exacerbations, indicating that MMP-9 is an important inflammatory mediator, which caused airway inflammation and acute exacerbations. The present study also indicated that the MMP-9 levels and the MMP-9/TIMP-1 ratio were negatively correlated with the use of bronchodilators, but that TIMP-1 was positively correlated with the use of bronchodilators, indicating that a modest increase of bronchodilators in COPD patients may be beneficial for controlling airway inflammation and airway remodeling mediated by MMP-9 and reducing the frequency of acute exacerbations of COPD.

In the current study, the level of MMP-9 in patients without emphysema was markedly higher than patients with emphysema (although this difference was statistically insignificant). However, the FEV1\% pred of the patients with COPD without 
was better than those patients with mild emphysema. This indicated that MMP-9 may be partly involved in the type of airway inflammation and airway remodeling of patients with COPD.

In the present study, the expression of MMP-9 in the COPD patients with severe emphysema was obviously higher than that in the mild emphysema group, suggesting that MMP-9 is not only involved in the progress of COPD, but that it is also associated with the severity of emphysema.

A study by Hong et al (24) indicated that among COPD patients, the small airway wall thickness of non-smoking females was thicker than that of non-smoking males, whereas the condition of emphysema was better in female than in male patients. Of note, non-smoking female patients were more likely to exhibit bronchial wall thickening and less likely to exhibit emphysema. In the present study, the proportion of non-smoking andfemale patients in the non-emphysema group was higher compared with that in the emphysema groups.

A study by Kim et al (25) revealed that patients with the bronchitis type of COPD exhibited more clinical symptoms and acute exacerbations; furthermore, the $\mathrm{PaO}_{2}$ in the non-emphysema group was lower than that in the emphysema group, and although there was no statistically significant difference, more clinical symptoms appeared, which was associated with the airway inflammation, indicating that the repeated outbreak of chronic airway inflammation was an important source of further clinical symptoms and acute exacerbations. In addition, compared with that in the COPD patients who exhibited different degrees of emphysema, the dosage of intravenous glucocorticoids in the non-emphysema patients was much lower. A correlation analysis indicated that the stage of emphysema was negatively correlated with bronchodilators in patients with COPD. The possible reason was that the airway stenosis and mucus secretion were more obvious in the non-emphysema patients who required using more bronchodilators and mucolytics.

Studies have indicated that comorbidities have a great influence on the quality of life as well as the frequent acute exacerbations and death $(9,25)$; furthermore, different types of comorbidities had significantly different effects on the relative risk of death, which was much higher in the COPD patients who exhibited combinations of tumors, cardiovascular disease, depressive disorders, pulmonary interstitial fibrosis and cirrhosis. The present study also suggested that the mortality rate in patients with a COTE index of $\geq 4$ points was 2.2 times higher than that in patients whose COTE index ranged from 0 to 3 points. The common comorbidities, including pulmonary heart disease, coronary heart disease, various types of cardiovascular disease (e.g., hypertension and atrial fibrillation), as well as various types of respiratory failures and heart failure, were analyzed in the present study. The level of MMP-9 was significantly increased in the COPD patients who had $>3$ comorbidities and a COTE index of $\geq 4$ points, illustrating that persistent inflammation in the peripheral blood may be an important factor for acute attack and the severity of comorbidieties. Furthermore, it was revealed that in the COPD patients with various cardiovascular diseases, the number of non-emphysema patients was greater than the number of emphysema patients; however, there was no statistically significant difference among the different severities of emphysema. Previous studies on COPD patients with atrial fibrillation demonstrated that recurrent episodes of systemic and local chronic airway inflammation, as well as continuous increases of inflammatory mediators in the circulating blood, are important factors leading to arteriosclerosis and increased cardiovascular events caused by the dysfunction of vascular endothelium (26-28). In the present study, the levels of MMP-9 in COPD patients with cardiovascular disease were higher than those in patients without cardiovascular disease. A correlation analysis indicated that MMP-9 was positively correlated with the quantity of comorbidities and the COTE index, and particularly correlated with cardiovascular disease and coronary heart disease. It also indicated that elevated MMP-9 in the circulating blood of COPD patients was closely linked to cardiovascular disease.

A study by Wells et al (29) reported that pulmonary vascular disease was also associated with acute exacerbation of COPD. By using chest CT to measure the pulmonary artery to aorta ratio (PA:A), as was presented in Fig. 3. The main pulmonary artery (PA) and aortic (A) diameters at the level of the bifurcation were used to calculate the PA:A ratio. In cases where A was not uniform in diameter, two measurements were taken 90 degrees apart and the larger diameter was used. Panel B presents the spatial association of PA with A. Panel $\mathrm{C}$ is overlaid on the axial CT image (Fig. 3). The study revealed that COPD patients with a PA:A of $>1$ were more likely to develop severe respiratory and sleep disorders, pulmonary embolism, hypoxemia and cardiac insufficiency. No statistically significant difference in the number of COPD patients with pulmonary heart diseasebetween the non-emphysema and severe emphysema groups was identified, but there were significantly more patients who exhibited pulmonary heart diseasein the mild emphysema group than in the other groups. MMP-9 in the circulating blood was not correlated with the presence of pulmonary heart disease. Furthermore, the study indicated that COPD patients with pulmonary heart disease were not only impacted by MMP-9, but also by other contributing factors.

The present study was performed only for the purposes of clinical research for one hospital. Although several inflammatory factors were involved in the pathogenetic process of COPD, only MMP-9 and TMP-1 were selected and emphasized in the current study. Therefore, further studies are required to verify these results.

In conclusion, MMP-9 is mainly involved in the pathogenesis of COPD and is correlated with acute exacerbationsand the number of comorbidities. It was indicated that MMP-9 and the MMP-9/TIMP-1 ratio could possibly involve in the pathogenesis of differentemphysematypes of COPD patients and may have a critical role in various comorbidities of COPD.

\section{Acknowledgements}

Not applicable.

\section{Funding}

No funding received. 


\section{Availability of data and materials}

The datasets generated and/or analyzed during the current study are not publicly available due to insuffient funding, but are available from the corresponding author on reasonable request.

\section{Authors' contributions}

ZL designed and implemented the current study, and wrote the manuscript. FS, J-XL and C-LG collected and sorted cases. M-MP, JL translated the manuscript and analyzed statistical data. P-XL advised and instructed the current study.

\section{Ethics approval and consent to participate}

The present study was authorized by the Ethics Committee of the Petroleum Clinical Medical College of Hebei Medical University. All patients who were enrolled in this study had provided their informed consent.

\section{Patient consent for publication}

Not applicable.

\section{Competing interests}

The authors declare that they have no competing interests regarding this study.

\section{References}

1. Ostridge K, Williams N, Kim V, Harden S, Bourne S Coombs NA, Elkington PT, Estepar RS, Washko G, Staples KJ and Wilkinson TM: Distinct emphysema subtypes defined by quantitative $\mathrm{CT}$ analysis are associated with specific pulmonary matrix metalloproteinases. Respir Res 17: 92, 2016.

2. Papakonstantinou E, Karakiulakis G, Batzios S, Savic S, Roth M, Tamm M and Stolz D: Acute exacerbations of COPD are associated with significant activation of matrix metalloproteinase 9 irrespectively of airway obstruction, emphysema and infection. Respir Res 16: 78, 2015.

3. Smith BM, Austin JH, Newell JD Jr, D'Souza BM, Rozenshtein A, Hoffman EA, Ahmed F and Barr RG: Pulmonary emphysema subtypes on computed tomography: The MESA COPD study. Am J Med 127: 94.e7-e23, 2014.

4. Foster WL Jr, Pratt PC, Roggli VL, Godwin JD, Halvorsen RA Jr and Putman CE: Centrilobular emphysema: CT-pathologic correlation. Radiology 159: 27-32, 1986.

5. Hruban RH, Meziane MA, Zerhouni EA, Khouri NF, Fishman EK, Wheeler PS, Dumler JS and Hutchins GM: High resolution computed tomography of inflation-fixed lungs. Pathologic-radiologic correlation of centrilobular emphysema. Am Rev Respir Dis 136: 935-940, 1987.

6. Montaño M, Sansores RH, Becerril C, Cisneros J, González-Avila G, Sommer B, Ochoa L, Herrera I, Ramírez-Venegas A and Ramos C: FEV1 inversely correlates with metalloproteinases 1, 7, 9 and CRP in COPD by biomass smoke exposure. Respir Res 15: 74, 2014.

7. Grzela K, Litwiniuk M, Zagorska W and Grzela T: Airway remodeling in chronic obstructive pulmonary disease and asthma: The role of matrix metalloproteinase-9. Arch Immunol Ther Exp (Warsz) 64: 47-55, 2016.

8. Jia TG, Zhao JQ and Liu JH: Serum inflammatory factor and cytokines in AECOPD. Asian Pac J Trop Med 7: 1005-1008, 2014.

9. Divo M,Cote C, de Torres JP,Casanova C,Marin JM,Pinto-Plata V, Zulueta J, Cabrera C, Zagaceta J, Hunninghake G, et al: Comorbidities and risk of mortality in patients with chronic obstructive pulmonary disease. Am J Respir Crit Care Med 186: $155-161,2002$
10. Celli BR and MacNee W; ATS/ERS Task Force: Standards for the diagnosis and treatment of patients with COPD: A summary of the ATS/ERS position paper. Eur Respir J 23: 932-946, 2004.

11. Kitaguchi Y, Fujimoto K, Kubo K and Honda T: Characteristics of COPD phenotypes classified according to the findings of HRCT. Respir Med 100: 1742-1752, 2006.

12. Roberts HR, Wells AU, Milne DG, Rubens MB, Kolbe J, Cole PJ and Hansell DM: Airflow obstruction in bronchiectasis: Correlation between computed tomography features and pulmonary function tests. Thorax 55: 198-204, 2000.

13. The definition of emphysema. Report of a National Heart, Lung, and Blood Institute, Division of Lung Diseases workshop. Am Rev Respir Dis 132: 182-185, 1985.

14. Hoffman EA, Simon BA and Mclennan G: State of the art. A structural and functional assessment of the lung via multidetector-row computed tomography: Phenotyping chronic obstructive pulmonary disease. Proc Am Thorac Soc 3: 519-532, 2006.

15. Singh S, Loke YK, Enright PL and Furberg CD: Mortality associated with tiotropium mist inhaler in patients with chronic obstructive pulmonary disease: Systematic review and meta-analysis of randomised controlled trials. BMJ 342: d3215, 2011.

16. Dar KA, Shahid M, Mubeen A, Bhargava R, Ahmad Z, Ahmad I and Islam $\mathrm{N}$ : The role of noninvasive methods in assessing airway inflammation and structural changes in asthma and COPD. Monaldi Arch Chest Dis 77: 8-18, 2012.

17. Kwiatkowska S, Noweta K, Zieba M, Nowak D and Bialasiewicz P: Enhanced exhalation of matrix metalloproteinase-9 and tissue inhibitor of metalloproteinase-1 in patients with COPD exacerbation: A prospective study. Respiration 84: 231-241, 2012.

18. Linder R, Rönmark E, Pourazar J, Behndig A, Blomberg A and Lindberg A: Serum metallopteinase-9 is related to COPD severity and symptoms-cross-sectional data from a population based cohort-study. Respir Res 16: 28, 2015.

19. Anderson AE Jr and Foraker AG: Centrerilobolar emphysema and panlobular emphysema: Two different diseases. Thorax 28: 547-550, 1973.

20. Saetta M, Kim WD, Izquierdo JL, Ghezzo H and Cosio MG: Extent of centriobular and panacinar emphysema in smokers'lungs: Pathological and mechanical implications. Eur Respir J 7: 664-671, 1994.

21. Kawayama T, Kinoshita T, Matsunaga K, Kobayashi A, Hayamizu T, Johnson M and Hoshino T: Responsiveness of blood and sputum inflammatory cells in Japanese copd patients, non-copd smoking controls, and non-copd nonsmoking controls. Int J Chron Obstruct Pulmon Dis 11: 295-303, 2016.

22. Segura-Valdezl L, Pardo A, Gaxiola M, Uhal BD, Becerril C and Selman M: Upregulation of gelatinases A and B, collagenases 1 and 2, and increased parenchymal cell death in COPD. Chest 117: 684-694, 2000

23. Linder R, Rönmark E, Pourazar J, Behndig A, Blomberg A and Lindberg A: Serum metalloproteinase-9 is related to COPD severity and symptoms-cross-sectional data from a population based cohort-study. Respir Res 16: 28, 2015.

24. Hong Y, Ji W, An S, Han SS, Lee SJ and Kim WJ: Sex differences of COPD phenotypes in nonsmoking patiens. Int J Chron Obstruct Pulmon Dis 11: 1657-1662, 2016.

25. Kim V, Han MK, Vance GB, Make BJ, Newell JD, Hokanson JE, Hersh CP, Stinson D, Silverman EK and Criner GJ; COPDGene Investigators: The chronic bronchitic phenotype of COPD: An analysis of the COPDGene study. Chest 140: 626-633, 2011.

26. Smith MC and Wrobel JP: Epidemiology and clinical impact of major comorbidities in patients with COPD. Int J Chron Obstruct Pulmon Dis 9: 871-888, 2014.

27. Selvarajah S, Todd I, Tighe PJ, John M, Bolton CE, Harrison T and Fairclough LC: Multiple Circulating cytokines are coelevated in chronic obstrictive pulmonary disease. Mediators Inflamm 2016: $3604842,2016$.

28. Eickhoff P, Valipour A, Kiss D, Schreder M, Cekici L, Geyer K, Kohansal R and Burghuber OC: Determinants of systemic vascular function in patients wity stable chronic obstructive pulmonary disease. Am J Respir Crit Care Med 178: 1211-1218, 2008.

29. Wells JM, Washko GR, Han MK, Abbas N, Nath H, Mamary AJ, Regan E, Bailey WC, Martinez FJ, Westfall E, et al: Pulmonary arterial enlargement and acute exacerbations of COPD. N Engl Med 367: 913-921, 2012. 